\title{
Efeitos das intempéries na rugosidade de painéis de partículas de Pinus sp.
}

\author{
Effects of weathering on the roughness of Pinus sp. \\ particleboards
}

\author{
Maria Fátima do Nascimento \\ André Luis Christoforo \\ Cristiane Inácio de Campos \\ Diego Henrique de Almeida \\ Francisco Antonio Rocco Lahr
}

\section{Resumo}

Maria Fátima do Nascimento Universidade de São Paulo São Carlos - SP - Brasil

André Luis Christoforo Universidade Federal de São Carlos São Carlos - SP - Brasil

Cristiane Inácio de Campos Universidade Estadual Paulista J úlio de Mesquita Filho Itapeva - SP - Brasil

Diego Henrique de Almeida Universidade Federal de São Carlos São Carlos - SP - Brasil

Francisco Antonio Rocco Lahr Universidade de São Paulo São Carlos - SP - Brasil

Recebido em 19/05/17 Aceito em 13/09/17

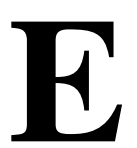

sta pesquisa objetivou avaliar a influência do período de exposição ao intemperismo ( $0,1,2$, 3 e 4 meses), do tipo de agente preservante

(CCA ou CCB) e do tipo de revestimento superficial (resina à base de óleo de mamona ou à base de água com filtro solar) nos valores da rugosidade (Ra) de painéis de partículas fabricados com madeira de Pinus sp. e resina poliuretana à base de mamona, assim como, investigar a possibilidade da estimativa da rugosidade em função dos valores dos parâmetros colorimétricos $\left(\mathrm{L}^{*}, \mathrm{a}^{*}\right.$ e b*). Os valores de rugosidade (entre 0,07 e 2,82 $\mu \mathrm{m}$ ) foram inferiores a 12,5 $\mu \mathrm{m}$, o que categoriza os painéis produzidos na classe N10 da norma ABNT NBR 8404 (1984), evidenciando assim, a potencialidade dos compósitos fabricados. O uso do revestimento com resina poliuretana forneceu os menores valores da rugosidade, além disso, as rugosidades dos painéis envelhecidos durante os quatro meses foram estatisticamente equivalentes, o que demonstra o bom desempenho dos materiais fabricados quanto às intempéries, e a boa qualidade obtida dos modelos de regressão multivariáveis $\left(\mathrm{R}^{2}=77,02 \%\right)$ evidenciam a possibilidade da estimativa da rugosidade por meio de parâmetros colorimétricos.

Palavras-chave: Painéis de partículas. Pinus sp. Rugosidade. Intemperismo natural. Colorimetria.

\section{Abstract}

The aim of this research was to evaluate the influence of the period of exposure $(0$, 1, 2, 3 and 4 months), the type of preservative agent (CCA and CCB) and the surface coating type (castor oil based polyurethane resin; water-based open pore coating with sunscreen) in the roughness values (Ra) of particleboards manufactured with wood Pinus sp.and castor oil based polyurethane resin, as well as to investigate the possibility of roughness estimation as a function of the measurements of colorimetric parameters $\left(L^{*}, a^{*}\right.$ and $\left.b^{*}\right)$. The results of the roughness values (between $0.07 \mu \mathrm{m}$ and $2.82 \mu \mathrm{m}$ ) were lower than $12.5 \mu \mathrm{m}$, which categorizes the panels produced in the N10 class of ABNT NBR 8404 (1984) standard, thus showing the potentiality of the composites manufactured. The use of the castor oil based polyurethane resin coating gave the lowest values of roughness and the roughness values of the aged panels during the four months were statistically equivalent, evidencing the good performance of the fabricated materials. The good quality obtained from the multivariable regression models $\left(R^{2}\right.$ $=77.02 \%$ ) show the possibility of estimating the roughness by colorimetric parameters.

Keywords: Particleboards. Pinus sp. Roughness. Natural weathering. Colorimetry. 


\section{Introdução}

A evolução do segmento industrial madeireiro trouxe diversos benefícios, podendo-se destacar a possibilidade de processamento da madeira e transformação de toras em vigas e tábuas de grandes dimensões. Entretanto, com o passar dos anos observou-se uma diminuição da disponibilidade de árvores de grande porte e o aumento do custo da madeira bruta. Tendo em vista esse cenário, o desenvolvimento dos painéis à base de madeira reconstituída, produzidos a partir de árvores de menores diâmetros, além da possibilidade de uso de resíduos de madeira já processada, mostraram-se viáveis e promissores (IRLE et al., 2012; BARBOSA et al., 2015; NASCIMENTO et al., 2016).

Os painéis à base de madeira apresentaram crescente desenvolvimento, principalmente no final do século XX. Acompanhando esse cenário, a indústria madeireira vem cada vez mais desenvolvendo técnicas para o uso mais eficiente da matéria-prima a partir de árvores de menores dimensões e resíduos madeireiros (AMERICAN..., 2006).

Dentre os resíduos madeireiros com potencialidades para produção de painéis destaca-se um grupo que, além da abundância, apresenta um problema ambiental quando descartados inadequadamente: os rejeitos e resíduos de madeira tratada, gerados em abundância no Brasil. Lepage (2010) avalia que a reutilização/reciclagem de madeira tratada reflete três aspectos importantes: conservação de florestas, diminuição do uso de aterros industriais e criação de mais oportunidades para o mercado da reciclagem. Esses rejeitos podem ser considerados como insumos de propriedades consideráveis no que diz respeito à resistência a biodegradação e possivelmente desempenho mecânico superior, conforme apontado por Lahr et al. (2011).

Questões ambientais referentes à utilização do arseniato de cobre cromatado (CCA), preservante hidrossolúvel de sais de cromo, cobre e arsênio, têm sido levantadas devido à dispersão do cobre e do arsênio para o ambiente antes da completa fixação dos ingredientes ativos, havendo a possibilidade de contaminação do solo e do lençol freático, principalmente na forma de resíduos, pois a pequena espessura dos resíduos aumenta a área passível de viabilizar a perda de substâncias por lixiviação (BERTOLINI et al., 2013). A possibilidade do aproveitando desse tipo de resíduos se apresenta como um desafio importante, e nesse contexto surge a possibilidade do uso de resíduos de madeira tratada com preservantes na produção dos painéis de partículas.
Nascimento et al. (2017) estudaram a rugosidade de painéis de partículas homogêneas produzidos com resíduos de madeira do gênero Pinus tratada e resinas poliuretana à base de óleo de mamona, e concluíram que o desempenho dos painéis apresentou classe de rugosidade adequada, sinalizando a viabilidade de uso desses resíduos na produção de painéis com matéria-prima tratada e sem o comprometimento da qualidade de colagem, ou seja, os resíduos de madeira tratada apresentaram boa interação com os adesivos utilizados.

Bertolini et al. (2013) estudou o uso de resíduos de madeira de Pinus tratados com borato de cobre cromatado (CCB) colados com resina poliuretana à base de óleo de mamona em painéis de partículas homogêneas e obteve resultados muito satisfatórios quanto às propriedades físicas, mecânicas e de envelhecimento, indicando a viabilidade de uso desse resíduo na produção dos painéis com excelente desempenho físico-mecânico.

Materiais que promovem melhores desempenhos físicos e mecânicos destacam-se na composição de um novo material. Nesse contexto, destacam-se os adesivos que garantem melhores propriedades na composição dos painéis de partículas. Zhang et al. (2011) estudaram o desempenho de adesivos compostos de ureia-formaldeído e polímero híbrido de epóxi-poliuretano (EPU), e obtiveram resultados excelentes quanto às propriedades mecânicas, térmicas e resistência à umidade em painéis particulados de palha.

Sulaiman et al. (2013) avaliaram as propriedades físicas e mecânicas de painéis de partículas de madeira produzidos com óleo de amido de palma modificado com epicloridrina, que consiste em um adesivo sustentável, e obtiveram resultados muito satisfatórios, indicando o uso desse compósito em aplicações comerciais. Resinas poliuretanas à base de óleo de mamona também têm sido utilizadas em pesquisas envolvendo painéis de partículas, e o uso desse adesivo se justifica por não ser tóxico, como é o caso de adesivos à base de ureia-formaldeído, e também pelas excelentes propriedades físicas e mecânicas proporcionadas aos painéis fabricados com essa resina (FERRO et al., 2014; BEZERRA et al., 2015; FREITAS et al., 2017).

$\mathrm{O}$ uso dos painéis à base de madeira vem conquistando espaço em diferentes aplicações dentro da construção civil. Inicialmente, foram usados como painéis de forro e divisórias. Evoluíram para o uso como pisos e também como telhas e materiais de cobertura. 
Uma das grandes preocupações para o uso dos painéis na construção civil refere-se à ação das intempéries sobre o material. Sendo assim, testes que avaliem o desempenho desses painéis quando expostos ao intemperismo norteiam e conduzem para diretrizes adequadas de um produto durável e competitivo (NASCIMENTO et al., 2016).

Os mecanismos de degradação estão diretamente relacionados com os agentes do meio ambiente, de forma isolada ou de forma conjunta. Os agentes climáticos causam maior efeito quando agem em conjunto. O principal mecanismo no processo de degradação em materiais poliméricos é a fotodeterioração, causada pela radiação ultravioleta somada à ação do calor, umidade e poluentes (BERTOLIINI et al., 2013).

Na madeira maciça, o principal sinal de degradação é sua descoloração superficial. Em seguida, observa-se o efeito global da fotodeterioração, fenômeno que, com o decorrer do tempo, afeta todo o material. As condições macroclimáticas, tais como temperatura, alterações climáticas e edáficas são os agentes de maior desgaste da madeira (TELES; COSTA, 2014). Por ser um material natural, a madeira exposta ao tempo e sem produtos que detenham os agentes biológicos é suscetível às condições ambientais. Tais considerações evidenciam que substâncias preservativas, produtos ignífugos e acabamentos superficiais devem ser incorporados ao material para maior durabilidade quando exposto às intempéries. A utilização da madeira (TELES; COSTA, 2014) e de produtos derivados (BERTOLINI et al., 2013) de maneira exposta e sem proteção à degradação causada pelo intemperismo possibilita a ocorrência de degradação que influenciam negativamente suas características físico-mecânicas.
Métodos alternativos têm sido utilizados na avaliação da madeira degradada, como é o caso do colorímetro, assim como discutido na pesquisa desenvolvida por Teles e Costa (2014). A variação das cores da madeira de angelim pedra antes e após o envelhecimento foi notada pela técnica de colorimetria em função das variáveis luminosidade $\left(\mathrm{L}^{*}\right)$ e pigmentos vermelho $\left(\mathrm{a}^{*}\right)$ e amarelo $\left(\mathrm{b}^{*}\right)$.

Em síntese, para a aplicação adequada da madeira e de produtos derivados em ambientes externos, como na forma de pisos, em que a rugosidade é uma das principais propriedades (NASCIMENTO et al., 2016), telhas (BOLAJI; MOSOBALAJE, 2008) entre outras, as propriedades dos materiais devem ser avaliadas na condição de exposição às intempéries (BERTOLINI et al., 2013).

O objetivo desta pesquisa é fabricar painéis de partículas com resíduos de madeira de Pinus sp. tratados com CCA e CCB, tratar a superfície com dois diferentes tipos de revestimentos (resina à base de mamona e um produto comercial), expô-los ao intemperismo natural $(0,1,2,3$ e 4 meses), determinar a rugosidade da superfície $(\mathrm{Ra})$ e, além disso, avaliar a possibilidade da estimativa da rugosidade em função dos parâmetros colorimétricos ( $\mathrm{L}^{*}, \mathrm{a}^{*}$ e $\mathrm{b}^{*}$ ) obtidos com o uso do colorímetro.

\section{Material e métodos Produção dos painéis}

Para a produção de vinte painéis (um para cada tratamento - Quadro 1) foram utilizados resíduos de madeira de Pinus sp, tratados em autoclave com CCA e CCB, doados por uma empresa que atua no setor de preservação de madeira, situada na cidade de São Carlos, SP.

Quadro 1 - Tratamentos experimentais delineados

\footnotetext{
Nota: Legenda:

Trat. = Tratamento;

Rev. = Revestimento;

Int. = Tempo de exposição ao intemperismo;

$\mathrm{CCA}=$ Arseniato de Cobre Cromatado;

$\mathrm{CCB}=$ Borato de Cobre Cromatado;

$\mathrm{PU}=$ Resina poliuretana à base de mamona; e

$\mathrm{NL}=$ resina à base água para poro aberto.
}

\begin{tabular}{|c|c|c|c|c|c|c|c|}
\hline Trat. & Rev. & Pres. & Int. (meses) & Trat. & Rev. & Pres. & Int. (meses) \\
\hline 1 & PU & CCA & 0 & 11 & NL & CCA & 0 \\
\hline 2 & PU & CCA & 1 & 12 & NL & CCA & 1 \\
\hline 3 & PU & CCA & 2 & 13 & NL & CCA & 2 \\
\hline 4 & PU & CCA & 3 & 14 & NL & CCA & 3 \\
\hline 5 & PU & CCA & 4 & 15 & NL & CCA & 4 \\
\hline 6 & PU & CCB & 0 & 16 & NL & CCB & 0 \\
\hline 7 & PU & CCB & 1 & 17 & NL & CCB & 1 \\
\hline 8 & PU & CCB & 2 & 18 & NL & CCB & 2 \\
\hline 9 & PU & CCB & 3 & 19 & NL & CCB & 3 \\
\hline 10 & PU & CCB & 4 & 20 & NL & CCB & 4 \\
\hline
\end{tabular}


Para garantir a interação adequada entre as partículas utilizou-se resina poliuretana à base de óleo de mamona, bicomponente, com teor de sólidos de $100 \%$, sendo o componente A um poliol derivado de óleo vegetal, com densidade de 1,2 $\mathrm{g} / \mathrm{cm}^{3}$, e o componente B o isocianato polifuncional, com densidade de $1,24 \mathrm{~g} / \mathrm{cm}^{3}$. Esse adesivo apresenta como característica cura a frio, que pode ser acelerada com temperatura em torno de $100^{\circ} \mathrm{C}$.

Os resíduos foram obtidos na forma de maravalhas e passaram por um processamento em moinhomartelo do tipo Willye, da marca Marconi, modelo MA 680, utilizando peneira de abertura de 2,8 mm para obtenção das partículas. Optou-se pela utilização de partículas processadas em sua totalidade, utilizando também os finos obtidos no processamento. O uso das partículas menores justifica-se, pois elas auxiliam na melhor interação das partículas com a resina, proporcionando menores vazios nos painéis e também melhor acabamento superficial (CHRISTOFORO et al., 2016).

Para cada painel foram utilizados $1.400 \mathrm{~g}$ de partículas de Pinus sp. A massa utilizada de adesivo foi igual a $15 \%$ da massa de partículas. A mistura entre partículas e adesivos foi realizada em um encoladeira durante 5 minutos para homogeneização.

A pré-formação do colchão foi realizada em prensa mecânica com capacidade de 0,01 MPa. Em seguida a formação do painel foi realizada em prensa semiautomática da marca Marconi, modelo MA 098/50, com capacidade de carga de $800 \mathrm{kN}$ e temperatura máxima nos pratos de $200{ }^{\circ} \mathrm{C}$. Foi aplicada pressão de 4,0 MPa e temperatura de 100 ${ }^{\circ} \mathrm{C}$ por um período de 10 minutos, gerando painéis com dimensões de 400 mm x 400 mm e espessura nominal de $10 \mathrm{~mm}$ e densidade nominal de 0,85 $\mathrm{g} / \mathrm{cm}^{3}$.

Para a completa cura do adesivo, os painéis foram acondicionados por 72 horas em uma sala com temperatura ambiente e, posteriormente, esquadrejados em serra circular. Na sequência, os painéis foram lixados (com lixas de granulometria 100) para posterior aplicação do revestimento.

Os painéis foram revestidos com a resina poliuretana à base de óleo de mamona [PU] e com um produto comercial à base de água para poro aberto [NL], que penetra na madeira realçando os seus veios.

\section{Planej amento fatorial completo}

Os fatores e níveis experimentais avaliados na determinação dos valores da rugosidade [Ra] dos painéis foram o tipo de revestimento [Rev] (PU,
NL), o tipo de preservante [Pre] (CCA, CCB) e o número de meses aos quais os painéis revestidos ficaram expostos ao intemperismo natural [Int] $(0$, $1,2,3,4)$, resultando em um planejamento fatorial completo com 20 tratamentos, explicitados no Quadro 1. Deve ser ressaltado que o tempo 0 de exposição ao intemperismo denota a condição de referência, ou seja, os painéis que não foram expostos ao intemperismo

O planejamento estipulado não considerou a condição de painel sem tratamento presente, uma vez que não influenciou de forma significativa nas propriedades mecânicas dos painéis (BERTOLINI et al., 2014), e em todos os tratamentos o acabamento superficial (uso das resinas) foi considerado, por ser indispensável nas aplicações envolvendo pisos (VARANDA, 2016).

A influência dos três fatores e da interação entre eles nos valores da rugosidade dos painéis foi avaliada com o uso da análise de variância, considerada ao nível de 5\% de significância, e com o auxílio do software Minitab ${ }^{\circledR}$ versão 14 . A hipótese nula formulada $\left(\mathrm{H}_{0}\right)$ consistiu em se considerar equivalência entre as médias dos tratamentos, e a não equivalência das médias (pelo menos entre dois tratamentos) como hipótese alternativa $\left(\mathrm{H}_{1}\right)$. Pela formulação das hipóteses, Pvalor (probabilidade $\mathrm{P}$ ) superior ao nível de significância (P-valor $>0,05)$ implica que os 20 tratamentos delineados possuem médias estatisticamente equivalentes, e não equivalentes (pelo menos entre dois tratamentos) como hipótese alternativa.

O teste de Tukey, considerado ao nível de 5\% de significância, foi utilizado no agrupamento dos níveis do fator, de acordo com a análise de variância. No teste de Tukey, A denota o nível do fator associado ao maior valor médio, $\mathrm{B}$ consiste no nível do fator associado ao segundo maior valor médio, e assim sucessivamente, e letras iguais para níveis diferentes implicam médias estatisticamente equivalentes. Foram utilizados gráficos para a interpretação dos efeitos entre as interações dos fatores nos resultados obtidos para a rugosidade.

A rugosidade foi testada em 12 amostras para cada um dos 20 tratamentos experimentais delineados, resultando em 240 determinações.

\section{Intemperismo natural}

Após 72 horas da produção dos painéis (tempo de acondicionamento para cura do adesivo à base de mamona), eles foram cortados nas dimensões 100 $\mathrm{mm} \times 200 \mathrm{~mm}$ para serem colocados na estrutura preparada para exposição ao intemperismo natural. Antes de serem fixados na estrutura, os painéis 
foram revestidos com o próprio adesivo poliuretano à base de mamona e com o produto comercial (Figura 1).

Em seguida os painéis foram fixados com parafusos na estrutura de madeira preparada para exposição ao intemperismo natural (Figura 2).

Posteriormente à fixação dos painéis na estrutura de madeira, o conjunto foi encaminhado à área externa (Figura 3) para exposição às ações de intemperismo natural.

Os painéis revestidos ficaram expostos por 4 meses. As Figuras 4 e 5 ilustram os painéis, antes e após a exposição ao intemperismo. As medidas do colorímetro ( $\mathrm{L}^{*}, \mathrm{a}^{*}$ e $\mathrm{b}^{*}$ ) assim como as determinações da rugosidade foram obtidas antes da exposição ao intemperismo natural e também no final do primeiro, segundo, terceiro e quarto mês de exposição.

\section{Ensaio de rugosidade}

A rugosidade média foi obtida pela média aritmética dos valores absolutos das ordenadas de afastamento $\left(\mathrm{y}_{\mathrm{i}}\right)$ dos pontos do perfil de rugosidade em relação à linha média, dentro do percurso de medição. O ensaio para a determinação da rugosidade seguiu as recomendações da norma brasileira NBR 8404 (ABNT, 1984), utilizando corpos de prova com $350 \mathrm{~mm}$ de comprimento, largura variando entre $100 \mathrm{~mm}$ e $120 \mathrm{~mm}$ e espessura de $10 \mathrm{~mm}$. Os resultados dos valores da rugosidade obtidos nessa pesquisa foram comparados com os requisitos da norma brasileira e também com pesquisas correlatas.

Figura 1 - Painéis sem revestimento (a), sendo revestidos (b) e revestidos (c)

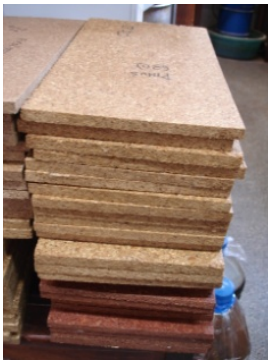

(a)

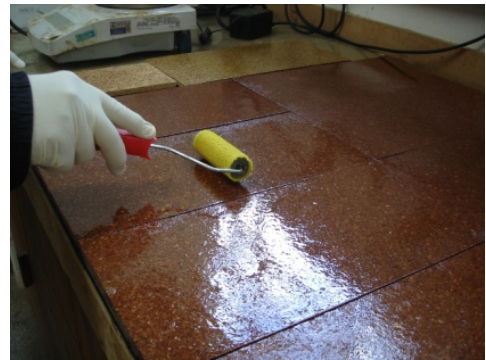

(b)

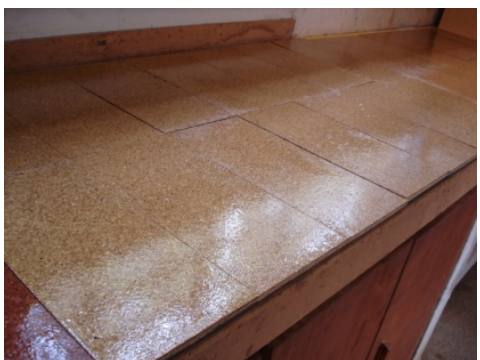

(c)

Figura 2 - Painéis sendo fixados (a), estrutura de madeira e painéis sobrepostos (b)

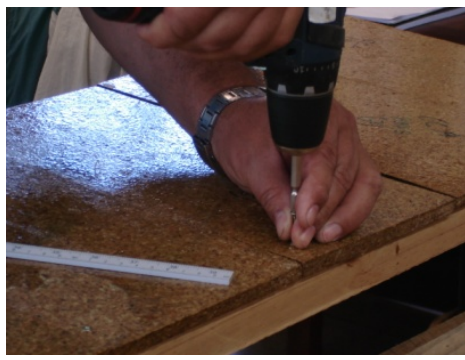

(a)

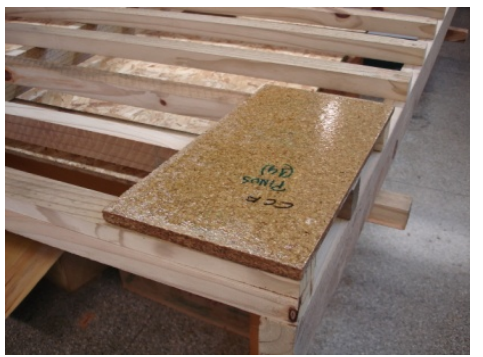

(b)

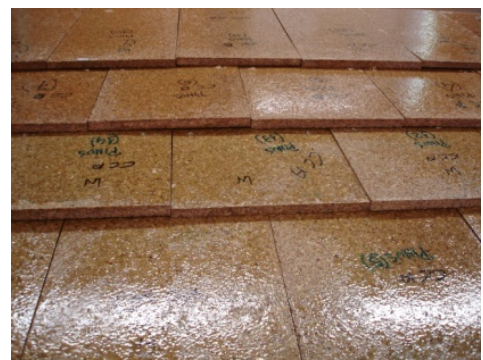

Figura 3 - Painéis expostos ao intemperismo natural

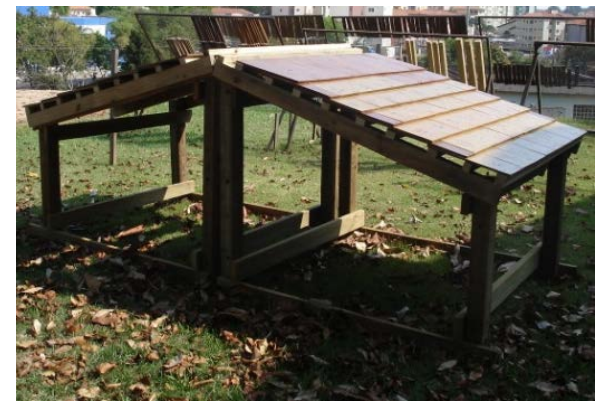


Figura 4 - Painéis revestidos com resina poliuretana à base de óleo de mamona antes (a) e após (b) os 4 meses de exposição

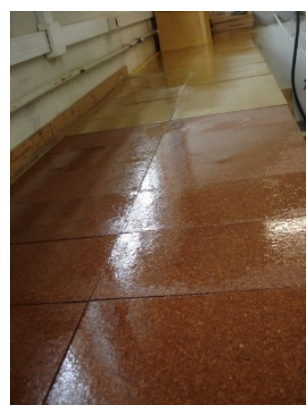

(a)

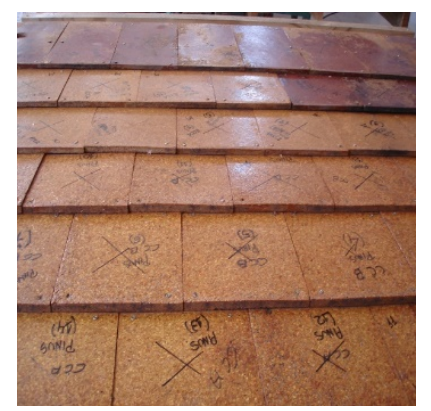

(b)

Figura 5 - Painéis recobertos com revestimento à base de água para poro aberto (NL) antes (a) e após (b) os 4 meses de exposição

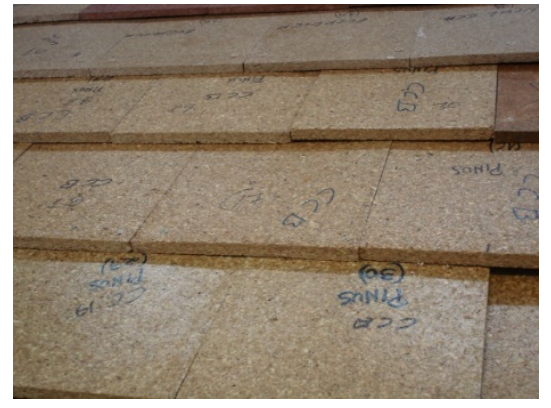

(a)

\section{Análise estatística}

Foram utilizados dois procedimentos estatísticos para a análise dos resultados. No primeiro, procurou-se investigar a influência dos fatores considerados nos valores da rugosidade dos painéis, avaliado por meio da análise de variância (ANOVA) do planejamento fatorial completo. No segundo, modelos de regressão multivariáveis baseados na ANOVA foram utilizados na tentativa de compreender os efeitos das variáveis exploradas do planejamento fatorial completo em cada uma das medidas obtidas com o auxílio do colorímetro (a, b e L), e posteriormente foi verificada a possibilidade da estimativa dos valores da rugosidade em função das medidas obtidas com o colorímetro.

\section{Modelos de regressão multivariáveis}

Dos testes realizados com o equipamento colorímetro, as variáveis obtidas foram a luminosidade $\left(\mathrm{L}^{*}\right)$ e os pigmentos com tons de vermelho $\left(a^{*}\right)$ e com tons de amarelo $\left(b^{*}\right)$.

Para cada um dos 20 tratamentos delineados, um painel foi escolhido aleatoriamente, e sobre sua superfície foram realizadas três medições das variáveis $L^{*}$, a* e b* com o uso do colorímetro portátil Konica Minolta, totalizando 60 determinações. Os valores obtidos para os

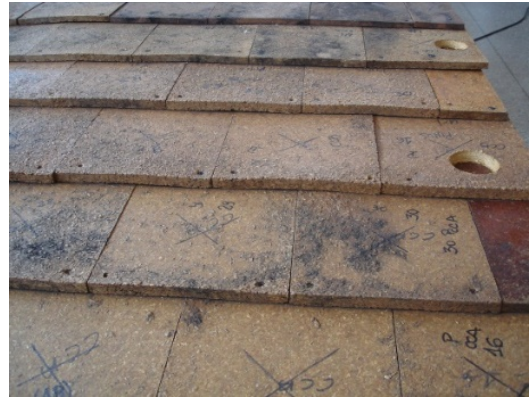

(b)

parâmetros colorimétricos consistiram na média das três medições realizadas.

Modelos de regressão multivariáveis foram utilizados para investigar a possibilidade de avaliar os efeitos dos fatores Rev, Pre e Int e das suas interações nos valores das medidas $\mathrm{L}^{*}, \mathrm{a}^{*}$ e $\mathrm{b}^{*}$ obtidas com o uso do colorímetro (Equação 1). Entendida a influência dos fatores e interações, outros modelos de regressão multivariáveis foram testados com o objetivo de relacionar as medias $\mathrm{L}^{*}$, $a^{*}$ e b* com os valores da rugosidade (Equação 2), permitindo avaliar a possibilidade de estimar os valores da rugosidade conhecendo-se os valores dos parâmetros colorimétricos.

$\mathrm{Y}=\beta_{0}+\beta_{1} \cdot \operatorname{Rev}+\beta_{2}$.Pre $+\beta_{3}$. Int $+\beta_{4} \cdot \operatorname{Rev} \cdot$ Pre + $\beta_{5} \cdot$ Rev $\cdot$ Int $+\beta_{6}$.Pre $\cdot$ Int $+\beta_{7}$.Rev $\cdot$ Pre $\cdot$ Int $+\varepsilon \quad$ Eq. 1

$\mathrm{Ra}=\alpha_{0}+\alpha_{1} \cdot \mathrm{L}^{*}+\alpha_{2} \cdot \mathrm{a}^{*}+\alpha_{3} \cdot \mathrm{b}^{*}+\alpha_{4} \cdot \mathrm{L}^{*} \cdot \mathrm{a}^{*}+$ $\alpha_{5} \cdot \mathrm{L}^{*} \cdot \mathrm{b}^{*}+\alpha_{6} \cdot \mathrm{a}^{*} \cdot \mathrm{b}^{*}+\alpha_{7} \cdot \mathrm{L}^{*} \cdot \mathrm{a}^{*} \cdot \mathrm{b}^{*}+\varepsilon \quad$ Eq. 2

Da Equação 1, Y assume os valores ora de $L^{*}$, de $a^{*}$ e de b*, e de ambas as Equações, $\beta_{\mathrm{i}}$ e $\alpha_{\mathrm{i}}$ são os coeficientes ajustados pelo método dos mínimos quadrados, sendo que o efeito da interação entre fatores é representado pelo produto das variáveis e $\varepsilon$ consiste no termo de erro aleatório obtido do ajuste.

Os modelos de regressão das Equações 1 e 2 foram avaliados com o uso da análise de variância, 
também ao nível de 5\% de significância, permitindo verificar a significância do modelo ajustado e também a significância de cada termo presente na equação. A qualidade do ajuste foi avaliada pelo coeficiente de determinação $\left(\mathrm{R}^{2}\right)$. Pela formulação das hipóteses, o modelo ajustado e os respectivos coeficientes $\left(\beta_{\mathrm{i}}\right.$ e $\alpha_{\mathrm{i}}$ ) são considerados significativos para $\mathrm{P}$-valores inferiores a 0,05 , e não significativos em caso contrário (P-valor $>0,05)$. Os fatores categóricos Rev e Pre foram ambos tratados como variáveis binárias (dummy). Para o revestimento, admitiu-se $\mathrm{Pu}=0$ e $\mathrm{NL}=1$, e para a variável tipo de preservante, admitiu-se $\mathrm{CCA}=0$ e $\mathrm{CCB}=1$.

\section{Resultados e discussão \\ Planejamento fatorial completo}

Na Tabela 1 são apresentados os valores médios, o coeficiente de variação e os menores e os maiores valores da rugosidade obtidos para cada um dos 20 tratamentos experimentais avaliados, e na Figura 6 são ilustrados os valores médios referentes aos 20 tratamentos.
Da Figura 6 nota-se a grande variabilidade nos valores médios da rugosidade decorrentes dos 20 tratamentos experimentais independentes, cujo menor $(0,07 \mu \mathrm{m})$ e o maior $(2,82 \mu \mathrm{m})$ valores de rugosidade foram provenientes dos tratamentos $1 \mathrm{e}$ 20 , respectivamente. Esses valores foram inferiores aos determinados por Rolleri e Roffael (2010) e Nascimento et al. (2017). Os resultados de rugosidade dos painéis produzidos nesta pesquisa foram inferiores a $12,5 \mu \mathrm{m}$, o que permite classificálos como pertencentes à classe $\mathrm{N} 10$ de acordo com a norma NBR 8404 (ABNT, 1984), evidenciando o bom desempenho dos materiais fabricados.

Na Tabela 2 são apresentados os resultados da ANOVA do planejamento fatorial completo sobre os valores da rugosidade dos painéis, sendo GL os graus de liberdade, SQ a soma dos quadrados, SQA a soma dos quadrados ajustada, MQA a média dos quadrados ajustada, F a estatística de Fisher, P-valor à probabilidade $\mathrm{P}$, e o símbolo $(x)$ denota a interação entre os fatores (dois a dois e dos três fatores conjuntamente).

Tabela 1 - Valores da rugosidade em função dos tratamentos delineados

\begin{tabular}{c|c|c|c|c}
\hline Tratamentos & $\begin{array}{c}\text { Média } \\
(\boldsymbol{\mu m})\end{array}$ & $\begin{array}{c}\text { Coeficiente } \\
\text { de Variação } \\
\mathbf{( \% )}\end{array}$ & $\begin{array}{c}\text { Valor } \\
\text { Mínimo }\end{array}$ & $\begin{array}{c}\text { Valor } \\
\text { Máximo }\end{array}$ \\
\hline 1 & 0,07 & 38,04 & 0,02 & 0,11 \\
2 & 0,28 & 64,17 & 0,10 & 0,56 \\
3 & 0,45 & 49,48 & 0,22 & 0,95 \\
4 & 0,38 & 62,29 & 0,21 & 0,98 \\
5 & 0,47 & 31,47 & 0,26 & 0,85 \\
6 & 0,11 & 65,34 & 0,01 & 0,26 \\
7 & 0,35 & 86,42 & 0,14 & 1,27 \\
8 & 0,49 & 49,41 & 0,22 & 1,02 \\
9 & 0,49 & 45,14 & 0,22 & 0,91 \\
10 & 0,49 & 31,30 & 0,33 & 0,79 \\
11 & 0,55 & 39,45 & 0,26 & 1,04 \\
12 & 2,00 & 23,30 & 1,25 & 2,85 \\
13 & 2,05 & 34,48 & 0,48 & 3,22 \\
14 & 1,93 & 44,42 & 0,74 & 3,55 \\
15 & 2,56 & 63,77 & 0,96 & 5,93 \\
16 & 0,11 & 65,34 & 0,01 & 0,26 \\
17 & 1,81 & 30,38 & 0,70 & 2,52 \\
18 & 1,72 & 51,05 & 0,48 & 3,05 \\
19 & 1,61 & 50,75 & 0,47 & 2,83 \\
20 & 2,82 & 61,09 & 0,47 & 6,10 \\
\hline
\end{tabular}


Figura 6 - Valores médios da rugosidade [Ra] para cada um dos Tratamentos estudados [Tr]

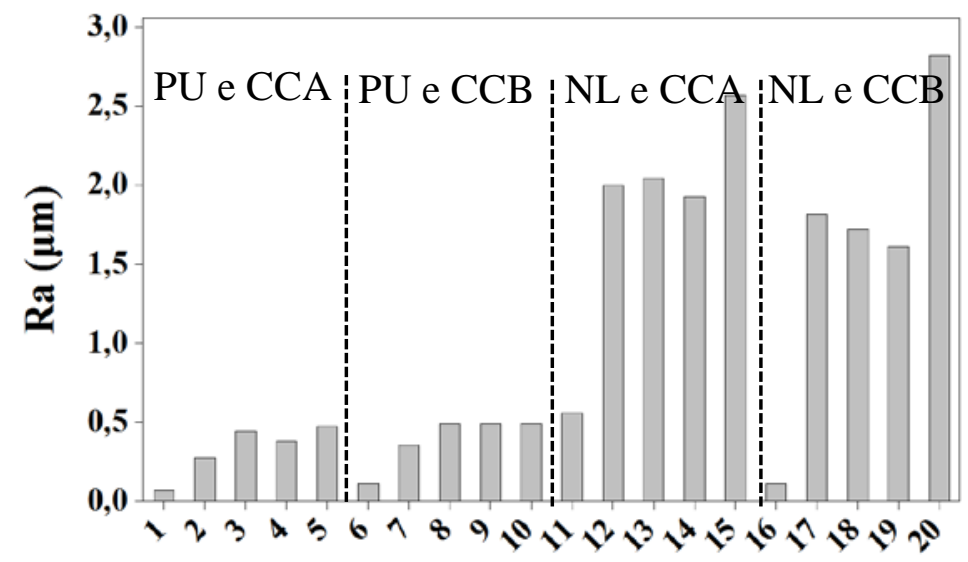

Tabela 2 - Resultados da ANOVA do planejamento fatorial completo sobre os valores da rugosidade dos painéis

\begin{tabular}{|c|c|c|c|c|c|c|}
\hline Fonte & GL & SQ & SQA & MQA & $\mathbf{F}$ & $\begin{array}{c}\text { P- } \\
\text { valor }\end{array}$ \\
\hline Revestimento & 1 & 110,7857 & 110,7857 & 110,7857 & 238,88 & 0,000 \\
\hline Preservante & 1 & 0,3125 & 0,3125 & 0,3125 & 0,67 & 0,413 \\
\hline Intemperismo & 4 & 48,6341 & 48,6341 & 12,1585 & 26,22 & 0,000 \\
\hline Revestimento $x$ Preservante & 1 & 1,0218 & 1,0218 & 1,0218 & 2,20 & 0,139 \\
\hline Revestimento $x$ Intemperismo & 4 & 24,3782 & 24,3782 & 6,0946 & 13,14 & 0,066 \\
\hline Preservativo $x$ Intemperismo & 4 & 0,9743 & 0,7943 & 0,1986 & 0,43 & 0,788 \\
\hline Revestimento $x$ Preservativo $x$ Intemperismo & 4 & 1,0151 & 1,0151 & 0,2538 & 0,55 & 0,701 \\
\hline Erro & 220 & 102,0276 & 102,0276 & 0,4638 & & \\
\hline Total & 239 & 288,9694 & & & & \\
\hline $\begin{array}{l}\text { Nota: Legenda: } \\
\qquad \begin{array}{l}\mathrm{GL}=\text { Graus de Liberdade; } \\
\mathrm{SQ}=\text { Soma dos Quadrados; } \\
\mathrm{SQA}=\text { Soma dos Quadrados Aj ustados; } \\
\mathrm{MQA}=\text { Média dos Quadrados Ajustada; e } \\
\mathrm{F}=\text { Estatística de Fisher; } \\
\text { P-valor = P-valor à probabilidade P. }\end{array}\end{array}$ & & & & & & \\
\hline
\end{tabular}

Da Tabela 2, apenas o tipo de revestimento [Rev] e o período de exposição dos painéis ao intemperismo [Int] foram considerados significativos sobre os valores da rugosidade dos painéis ( $\mathrm{P}$-valor $<0,05)$. $\mathrm{O}$ mesmo não ocorreu com o tipo de preservante [Pre] e nem com as interações dos fatores dois a dois e dos três fatores conjuntamente. Na Tabela 3 são apresentados os resultados do agrupamento pelo teste de Tukey dos níveis dos dois fatores considerados significativos.

Dentre os revestimentos utilizados nos painéis, a resina poliuretana à base de mamona forneceu os menores valores, $21 \%$ inferior ao valor médio da rugosidade dos painéis revestidos com NL. Com relação ao período de exposição ao intemperismo, os painéis da condição de referência (não expostos ao envelhecimento) forneceram os menores valores da rugosidade, $19 \%$ inferior ao valor médio da rugosidade dos painéis expostos a um mês ao intemperismo, e, como esperado, os maiores valores da $\mathrm{Ra}$ foram provenientes dos períodos de exposição ao intemperismo, mas independente do número de meses de exposição $(1,2,3,4)$. Os valores da rugosidade foram considerados estatisticamente equivalentes, o que evidencia a boa ação dos revestimentos. A Figura 7 apresenta os gráficos de efeitos principais do revestimento e do período de exposição ao intemperismo nos valores da rugosidade.

\section{Modelos de regressão}

Os valores médios das medidas L*, a* e b* obtidas do colorímetro para cada um dos 20 tratamentos experimentais são apresentados na Tabela 4. 


\section{Estimativa dos valores das medidas L*, a* e b* em função dos fatores Rev, Pre e Int}

Os modelos para a estimativa dos valores de $\mathrm{L}^{*}$, $\mathrm{a}^{*}$ e b* em função dos fatores Rev, Pre e Int e das interações entre esses fatores são apresentados nas Equações 3, 4 e 5, respectivamente, e os resultados da ANOVA do modelo de regressão são apresentados na Tabela 5. O modelo e os seus respectivos coeficientes são considerados significativos quando o P-valor é inferior ao nível de significância ( $\mathrm{P}$-valor $<0,05)$.

$\mathrm{L}^{*}=53,18+4,056 \cdot \operatorname{Rev}+3,266 \cdot$ Pre $-0,158 \cdot$ Int $4,404 \cdot \operatorname{Rev} \cdot$ Pre $-3,291 \cdot \operatorname{Rev} \cdot$ Int $-1,357 \cdot$ Pre $\cdot$ Int + $1,418 \cdot \operatorname{Rev} \cdot \operatorname{Pre} \cdot \operatorname{Int}\left[\mathrm{R}^{2}=93,48 \%\right]$

Eq. 3 $\mathrm{a}^{*}=5,778-1,438 \cdot \operatorname{Rev}-0,96 \cdot \operatorname{Pre}+0,117 \cdot \operatorname{Int}+$ $2,832 \cdot \operatorname{Rev} \cdot$ Pre $+0,683 \cdot \operatorname{Rev} \cdot$ Int $+0,614 \cdot$ Pre $\cdot$ Int $0,837 \cdot \operatorname{Rev} \cdot \operatorname{Pre} \cdot$ Int $\left[\mathrm{R}^{2}=82,20 \%\right] \quad$ Eq. 4 $\mathrm{b}^{*}=20,638-0,35 \cdot \operatorname{Rev}-0,536 \cdot \operatorname{Pre}-0,077 \cdot \operatorname{Int}+$

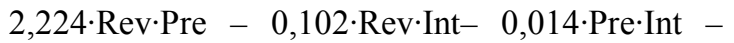
$1,284 \cdot \operatorname{Rev} \cdot \operatorname{Pre} \cdot \operatorname{Int}\left[\mathrm{R}^{2}=92,79 \%\right]$

Eq. 5

Os três modelos de regressão (Equações 3, 4 e 5) são considerados significativos pela ANOVA, e nota-se a boa precisão dos modelos em função dos valores dos coeficientes de determinação. Da Tabela 5, a variável $L^{*}$ é afetada de forma significativa por cinco dentre os oito termos componentes do modelo, e as variáveis a e b são afetadas de forma significativa por cinco e três termos, respectivamente. A exclusão dos termos não significativos nas Equações 3, 4 e 5 resultam nas Equações 6, 7 e 8, respectivamente.

$\mathrm{L}=52,864+3,32133 \cdot \operatorname{Rev}+2,53133 \cdot$ Pre $1,568 \cdot \operatorname{Rev} \cdot$ Pre $-2,92367 \cdot \operatorname{Rev} \cdot$ Int $-0,989667 \cdot$ Pre $\cdot$ Int [R $\left.\mathrm{R}^{2}=92,06 \%\right]$ Eq. 6 $\mathrm{a}^{*}=5,4388+0,7732 \mathrm{Rev} \cdot$ Pre $+0,433733 \cdot \operatorname{Rev} \cdot$ Int $+0,524067 \cdot$ Pre $\cdot$ Int $-0,3808 \cdot$ Rev $\cdot$ Pre $\cdot$ Int $\left[\mathrm{R}^{2}=76,56 \%\right.$ ]

Eq. 7

$\mathrm{b}^{*}=\quad 20,1113+1,86467 \cdot \mathrm{Rev} \cdot$ Pre $1.477 \cdot \operatorname{Rev} \cdot$ Pre $\cdot$ Int $\left[\mathrm{R}^{2}=87,76 \%\right] \quad$ Eq. 8

Os modelos reduzidos apresentados nas Equações 6, 7 e 8 também forneceram boas estimativas na determinação de L*, a* e b* em função dos bons valores dos coeficientes de determinação obtidos.

Com relação à Equação 6, nota-se que o termo independente é positivo $(52,864)$, e, dessa forma, o uso do revestimento e do preservante promovem aumentos nos valores da medida $L^{*}$, enquanto as iterações entre os fatores implicam na redução dessa variável (redução da luminosidade). Da Equação 7, a interação dos fatores dois a dois resultaram em aumentos no valor da medida $\mathrm{a}^{*}$, enquanto a interação entre os três fatores promoveu reduções nessa variável. O mesmo efeito foi constatado para a medida b (Equação 8).

\section{Estimativa dos valores da rugosidade em função das medidas do colorímetro}

O modelo de regressão que estabelece a relação entre a rugosidade [Ra] como função das medidas L*, a* e b* obtidas com o uso do calorímetro é expresso pela Equação 9, e os resultados da ANOVA do modelo de regressão são apresentados na Tabela 6.

$\begin{array}{lr}\mathrm{Ra}=406,45-6,37967 \cdot \mathrm{L}^{*} & -40,3387 \cdot \mathrm{a}^{*}- \\ 19,8846 \cdot \mathrm{b}^{*}+0,573434 \cdot \mathrm{L}^{*} \cdot \mathrm{a}^{*} & + \\ 0,313597 \cdot \mathrm{L}^{*} \cdot \mathrm{b}^{*}+2,0484 \cdot \mathrm{a}^{*} \cdot \mathrm{b}^{*} & - \\ 0,0296236 \cdot \mathrm{L}^{*} \cdot \mathrm{a}^{*} \cdot \mathrm{b}^{*}\left[\mathrm{R}^{2}=77,02 \%\right] & \text { Eq. } 9\end{array}$

$\mathrm{O}$ ajuste da Equação 9 forneceu valor de $\mathrm{R}^{2}=77,02 \%$, o que evidencia a possibilidade da estimativa dos valores da rugosidade em função das medidas L, a e b obtidas pelo colorímetro. Quatro dentre os oito termos do modelo foram considerados significativos pela ANOVA, e o modelo composto apenas pelos termos significativos é expresso pela Equação 10.

$\mathrm{Ra}=16,4478-0,238661 \cdot \mathrm{L}^{*}-0,330834 \cdot \mathrm{a} *-$ $0,0442362 \cdot b *-0,0010195 \cdot a * b *\left[R^{2}=71,26 \%\right]$

Eq. 10

Da Equação 10, aumentos nos valores das medidas $L^{*}, a^{*}, b^{*}$ e a*·b* promovem reduções nos valores da rugosidade dos painéis. O modelo reduzido também forneceu bom ajuste em função do valor do coeficiente de determinação obtido $\left(\mathrm{R}^{2}=71,26 \%\right)$.

Tabela 3 - Resultados do teste de Tukey dos fatores considerados significativos pela ANOVA sobre os valores de rugosidade [Ra]

\begin{tabular}{c|c|c|c|c|c|c|c}
\hline \multirow{2}{*}{ Propriedade } & \multicolumn{2}{|c|}{ Revestimento } & \multicolumn{5}{c}{ Intemperismo (meses) } \\
\cline { 2 - 8 } & PU & NL & $\mathbf{0}$ & $\mathbf{1}$ & $\mathbf{2}$ & $\mathbf{3}$ & $\mathbf{4}$ \\
\hline Rugosidade & B & A & B & A & A & A & A \\
\hline
\end{tabular}

Nota: Legenda:

$\mathrm{PU}=$ Resina poliuretana à base de mamona; $\mathrm{e}$

$\mathrm{NL}=$ resina à base água para poro aberto. 
Figura 7 - Gráficos de efeitos principais dos fatores considerados significativos sobre os valores da rugosidade

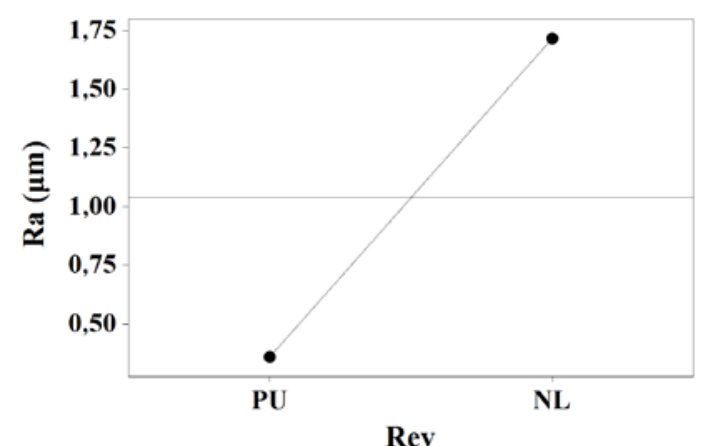

(a)

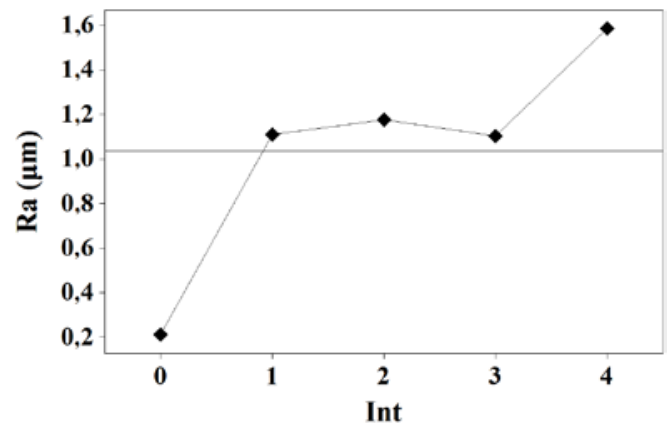

(b)

Tabela 4 - Valores médios das medidasL*, a* e b* para cada tratamento experimental delineado

\begin{tabular}{cccc|cccc}
\hline Tratamento & $\mathbf{L}^{*}$ & $\mathbf{a}^{*}$ & $\mathbf{b}^{*}$ & Tratamento & $\mathbf{L}^{*}$ & $\mathbf{a}^{*}$ & $\mathbf{b}^{*}$ \\
\hline 1 & 51,49 & 6,92 & 20,66 & 11 & 57,99 & 3,89 & 20,58 \\
2 & 54,48 & 4,81 & 20,85 & 12 & 52,68 & 5,34 & 19,85 \\
3 & 53,96 & 5,43 & 20,17 & 13 & 50,55 & 6,45 & 19,74 \\
4 & 52,90 & 5,98 & 20,08 & 14 & 46,77 & 6,92 & 19,74 \\
5 & 51,49 & 6,92 & 20,66 & 15 & 43,70 & 7,10 & 19,74 \\
6 & 58,06 & 4,61 & 20,63 & 16 & 56,86 & 5,91 & 21,85 \\
7 & 53,00 & 5,90 & 19,73 & 17 & 52,20 & 6,81 & 20,47 \\
8 & 52,97 & 6,25 & 19,47 & 18 & 48,75 & 7,88 & 19,55 \\
9 & 52,13 & 6,85 & 19,46 & 19 & 45,56 & 8,06 & 17,08 \\
10 & 50,92 & 7,79 & 20,31 & 20 & 43,24 & 8,17 & 16,16 \\
\hline
\end{tabular}

Tabela 5 - Resultados da ANOVA dos modelos de regressão para a estimativa dos valores de L*, a* e b*

\begin{tabular}{l|c|c|c}
\hline \multicolumn{1}{c|}{ Termos } & L* $^{*}$ (P-valor) & $\mathbf{a}^{*}-(\mathrm{P}$-valor $)$ & $\mathbf{b}^{*}-$ (P-valor) \\
\hline Constante & 0,000 & 0,000 & 0,000 \\
Revestimento & 0,010 & 0,058 & 0,466 \\
Preservante & 0,031 & 0,187 & 0,271 \\
Intemperismo & 0,690 & 0,566 & 0,577 \\
Revestimento $x$ Preservante & 0,038 & 0,013 & 0,005 \\
Revestimento $x$ Intemperismo & 0,000 & 0,031 & 0,601 \\
Preservativo $x$ & 0,029 & 0,049 & 0,942 \\
Intemperismo & 0,091 & 0,046 & 0,000 \\
Revestimento $x$ Preservativo $x$ Intemperismo & 0,000 & 0,000 & 0,000 \\
\hline Modelo & & &
\end{tabular}

Tabela 6 - Resultados da ANOVA do modelo de regressão para a estimativa dos valores da rugosidade em função das medidas obtidas pelo calorímetro

\begin{tabular}{l|c}
\hline \multicolumn{1}{c|}{ Termos } & Ra $-(\mathrm{P}$-valor $)$ \\
\hline Constante & 0,272 \\
$\mathrm{~L}^{*}$ & 0,001 \\
$\mathrm{a}^{*}$ & 0,022 \\
$\mathrm{~b}^{*}$ & 0,013 \\
$\mathrm{~L}^{*} x \mathrm{a}^{*}$ & 0,080 \\
$\mathrm{~L}^{*} x \mathrm{~b}^{*}$ & 0,095 \\
$\mathrm{a}^{*} x \mathrm{~b}^{*}$ & 0,045 \\
$\mathrm{~L}^{*} x \mathrm{a}^{*} x \mathrm{~b}^{*}$ & 0,087 \\
\hline Modelo & 0,000 \\
\hline
\end{tabular}




\section{Conclusões}

Os resultados obtidos da presente pesquisa possibilitam concluir que:

(a) dentre as resinas utilizadas, o revestimento à base de água para poro aberto forneceu os maiores valores de rugosidade, e, com relação ao intemperismo, a não exposição dos materiais (condição de referência) forneceu os menores valores da rugosidade, assim como esperado; entretanto, os períodos de exposição de 1 a 4 meses dos materiais foram considerados equivalentes, evidenciando o bom desempenho dos painéis fabricados;

(b) os bons resultados obtidos dos coeficientes de determinação $(82,20 \%$ a 93,48\%) dos modelos de regressão para a estimativa dos valores $L^{*}, a^{*}$ e b* do colorímetro em função dos fatores revestimento, preservante, intemperismo e da interação entre eles evidenciaram a possibilidade da estimativa dos parâmetros colorimétricos; e

(c) a boa qualidade do ajuste obtido $\left(\mathrm{R}^{2}=77,02 \%\right)$ do modelo de regressão para a estimativa da rugosidade em função das medidas $L^{*}$, a* e b* do colorímetro evidencia a possibilidade da estimativa dessa propriedade apenas pela leitura do aparelho, o que se apresenta como metodologia promissora na obtenção de propriedades de painéis de partículas.

\section{Referências}

AMERICAN FOREST AND PAPER ASSOCIATION. Engineered Wood Products Primer Awareness Guide. Washington: American Wood Council, 2006. Disponível em: $<$ http://www.woodaware.info/PDFs/EWPPrimer.p df $>$. Acesso em: 30 maio 2011.

\section{ASSOCIAÇÃO BRASILEIRA DE NORMAS} TÉCNICAS. NBR 8404: indicação do estado de superfícies em desenhos técnicos. Rio de Janeiro, 1984.

BARBOSA, J. C. et al. Medium Density Particleboard Reinforced With Bamboo Laminas.

BioResources, v. 10, p. 330-335, 2015.

BERTOLINI, M. S. et al. Accelerated Artificial Aging of Particleboards From Residues of CCB TREATED Pinus sp. and Castor Oil Resin. Materials Research, São Carlos, v. 16, p. 293303, 2013.

BERTOLINI, M. S. et al. Painéis de Partículas Provenientes de Rejeitos de Pinus sp. Tratado Com Preservante CCA e Resina Derivada de Biomassa. Revista Árvore, v. 38, p. 339-346, 2014.
BEZERRA, B. S. et al. Physical and Mechanical Characteristic of PARTICLEBOARDS Produced With Residues of Sugarcane and Stem Leaves of Bamboo Bonded With Castor Oil Adhesive.

Advanced Materials Research, v. 1088, p. 652655, 2015.

BOLAJI, A.; MOSOBALAJE, O. A. Development of a Roofing Material From Polyethylene Through the Use of Additives. Journal of Materials in Civil Engineering, v. 20, n. 8, 2008.

CHRISTOFORO, A. L. et al. Homogeneous Pinus sp. Particleboards Reinforced With Laminated Composite Materials. Engenharia Agrícola, v. 36, p. 558-565, 2016.

FERRO, F. S. et al. Influence of Proportion Polyol/Pre-Polymer Castor Oil Resin Components in Static Bending Properties of Particleboards Produced With Pinus sp. Advanced Materials Research, v. 884-885, p. 667-670, 2014.

FREITAS, J. F. et al. A Preliminary Study About the Utilization of Cajueiro and Amescla for MDP Panels Production. International Journal of Materials Engineering, v. 7, p. 21-24, 2017.

IRLE, M. A. et al. Wood Composites. In: ROWELL, R. M. Handbook of Wood Chemistry and Wood Composites. Boca Raton: CRC Press, 2012.

LAHR, F.A. R. et al. Qualidade de Chapas de Partículas de Pinus elliottii Colocadas Com Resina Poliuretana Sob Diferentes Combinações de Pressão e Temperatura. Ciência Florestal, Santa Maria, v. 21, n. 3, p. 551-558, 2011.

LEPAGE, E. S. Moq ${ }^{\circledR} 50$ 0x (CCB): um preservativo sustentável com um longo histórico de eficiência. In: ENCONTRO BRASILEIRO EM MADEIRAS E EM ESTRUTURAS DE MADEIRA, 12., Lavras, 2010. Anais.. Lavras: UFLA, IBRAMEM, 2010.

NASCIMENTO, M. F. et al. Painéis de Partículas Homogêneas Fabricados Com Resíduos Lignoceluósicos e Resina Alternativa Para Aplicação em Pisos. Scientia Forestalis, v. 44, p. 1-8, 2016.

NASCIMENTO, M. F. et al. Roughness Study on Layer Panels Manufactured Form Treated Wood Waste. Acta Scientiarum. Technology, v. 39, n. 1, p. 27-32, 2017.

ROLLERI, A.; ROFFAEL, E. Surface Roughness of Uncoated Particleboards and Its Relation With the Raw Material, Adhesive and Climatic Conditions. European Journal of Wood and Wood Products, v. 68, n. 4, p. 369-372, 2010. 
SULAIMAN, N. S. et al. Evalution of the Properties Made Using Oil Palm Starch Modified With Epichlorohydrin. BioResources, v. 8, n. 1, p. 283-30, 2013.

TELES, R. F.; COSTA, A. F. Influência do Intemperismo Acelerado nas Propriedades Colorimétricas da Madeira de Angelim Pedra. Nativa, v. 2, n. 2, p. 65-70, 2014.
VARANDA, L. D. Painéis de Alta Densidade Para Aplicação em Pisos: produção e avaliação de desempenho. São Carlos, 2016. Tese (Doutorado em Engenharia) - Programa de PósGraduação em Ciências e Engenharia de Materiais, Universidade de São Paulo, São Carlos, 2016.

ZHANG, Y. et al. Straw Based Particleboard Bonded With Composite Adhesives.

BioResources, v. 6, n.1, p. 646-476, 2011.

Maria Fátima do Nascimento

Laboratório de Madeiras e de Estruturas de Madeira, Escola de Engenharia | Universidade de São Paulo | Av. Trabalhador Sãocarlense, 400, Centro | São Carlos - SP - Brasil | CEP 13566-590 | Tel.: (16) 3373-8206 | E-mail: fati@sc.usp.br

\section{André Luis Christoforo}

Departamento de Engenharia Civil | Universidade Federal de São Carlos | Rodovia Washington Luís, km 235, SP-310 | São Carlos - SP Brasil | CEP 36307-352 | Tel.: (16) 3351-8262 | E-mail: christoforoal@yahoo.com.br

\section{Cristiane Inácio de Campos}

Câmpus Experimental de Itapeva | Universidade Estadual Paulista J úlio de Mesquita Filho | Rua Geraldo Alckimin, 519, Vila Nossa Senhora de Fátima | Itapeva - SP - Brasil | CEP 18409-010 | Tel.: (15) 3524-9131 | E-mail: cristiane@itapeva.unesp.br

\section{Diego Henrique de Almeida}

Departamento de Engenharia Civil | Universidade Federal de São Carlos | E-mail: diegoestruturas@gmail.com

\section{Francisco Antonio Rocco Lahr}

Escola de Engenharia | Universidade de São Paulo | E-mail: frocco@sc.usp.br

\section{Revista Ambiente Construído}

Associação Nacional de Tecnologia do Ambiente Construído

Av. Osvaldo Aranha, 99 - 3o andar, Centro

Porto Alegre - RS - Brasil

CEP $90035-190$

Telefone: +55 (51) 3308-4084

Fax: +55 (51) 3308-4054

www. seer. ufrgs. br/ ambienteconstruido

E-mail: ambienteconstruido@ufrgs.br

This is an open-access article distributed under the terms of the Creative Commons Attribution License. 